\title{
LETTERS \\ Is Training in a Primary Care Internal Medicine Residency Associated with a Career in Primary Care Medicine?
}

\author{
Sudeep K. Aulakh, MD, FACP, FRCPC, Gina L. Luciano, MD, and Michael J. Rosenblum, MD \\ Department of Medicine, Baystate Medical Center/Tufts University School of Medicine, Springfield, MA, USA.
}

J Gen Intern Med 31(3):268

DOI: $10.1007 / \mathrm{s} 11606-015-3542-9$

(c) Society of General Internal Medicine 2015

$\mathrm{T}$ o the Editor- We wholeheartedly agree with Stanley et. al. ${ }^{1}$ about how the residency experience seems to dissuade residents from pursuing primary care careers, and agree that graduate medical education reform is imperative. As the authors describe, there are many reasons why this is the case. Training programs overwhelmingly favor hospital-based rotations, with outpatient medicine typically representing not more than one-third of training time. Simply increasing ambulatory time does not address the challenges that discourage residents from pursuing primary care careers. Additionally, many residency clinics provide care to socioeconomically disadvantaged patients with complex needs. $^{2}$ Expecting trainees to be confident and fully engaged in ambulatory care with our current training models is unrealistic.

We have designed a primary care training program to address these challenges, with $>50 \%$ of the training in the ambulatory environment. To prepare primary care residents for complex disease management in an ambulatory setting, our residents participate in year-long longitudinal subspecialty ambulatory electives with subspecialty preceptors, as opposed to hospital-based subspecialty electives. ${ }^{3}$ These subspecialty preceptors have become resources for our residents, allowing them to experience the importance of teamwork and collaboration in primary care. We have implemented a second primary care continuity site to expose residents to diverse patient populations and ambulatory environments.

To preserve the passion for advocacy that residents possess, we have integrated a community project into our program. Protected time allows residents to pursue advocacy projects of their choice. Examples of projects include a Spanish/English language and cultural exchange program, a high school nutrition curriculum, promoting health education in a homeless shelter and volunteering services to a local free clinic. Each self-selected project helps to keep our residents grounded in what initially attracted them to primary care.

Mentorship is an essential feature of our program. Just as residents are encouraged to pursue subspecialty careers, it is equally important to enthusiastically encourage primary care careers. Quarterly get-togethers with the primary care codirectors are instrumental in maintaining engagement. During these dinners, we discuss medical, political and cultural issues in primary care, facilitate dialogue about career goals and promote peer group mentoring.

We have graduated two classes of primary care residents to date, and $80 \%$ of our graduates have pursued primary care careers. Additionally, several categorical residents have transferred into our program, while none of the primary care residents have left. We believe that continued innovation and mentorship will help to successfully build and maintain resident interest in primary care.

Corresponding Author: Sudeep K. Aulakh, MD, FACP, FRCPC; Department of Medicine, Baystate Medical Center/Tufts University School of Medicine, Springfield, MA, USA (e-mail: Sudeep.Aulakh@bhs.org).

\section{REFERENCES}

1. Stanley M, O'Brien B, Julian K, Jain S, Cornett P, Hollander H, Baron RB, Kohlwes RJ. Is training in a primary care internal medicine residency associated with a career in primary care medicine? J Gen Intern Med. 2015;30:1333-1338.

2. Nadkarni M, Reddy S, Bates CK, Fosburgh B, Babbott S, Holmboe E. Ambulatory-based education in internal medicine: current organization and implications for transformation. Results of a national survey of resident continuity clinic directors. J Gen Intern Med. 2011;26:16-20.

3. Luciano G, Rosenblum M, Aulakh S. Training the ambulatory internist: rebalancing residency education. Med Educ. 2014;48:535-536. 